\title{
A binary particle swarm optimization approach for power system security enhancement
}

\author{
Padmanabha Raju Chinda, Ragaleela Dalapati Rao \\ Department of Electrical and Electronics Engineering, Prasad V. Potluri Siddhartha Institute of Technology, Vijayawada, India
}

\begin{tabular}{|c|c|}
\hline Article Info & ABSTRACT \\
\hline Article history: & Improvement of power system security manages the errand of making \\
\hline Received Feb 18, 2021 & $\begin{array}{l}\text { healing move against conceivable system overloads in the framework } \\
\text { following the events of contingencies. Generation re-dispatching is answer }\end{array}$ \\
\hline Revised May 28, 2021 & for the evacuation of line overloads. The issue is the minimization of \\
\hline Accepted Jul 19, 2021 & $\begin{array}{l}\text { different goals viz. minimization of fuel cost, minimization of line loadings } \\
\text { and minimization of overall severity index. Binary particle swarm }\end{array}$ \\
\hline Keywords: & $\begin{array}{l}\text { optimization (BPSO) method was utilized to take care of optimal power flow } \\
\text { issue with different targets under system contingencies. The inspiration to }\end{array}$ \\
\hline $\begin{array}{l}\text { Binary particle swarm } \\
\text { optimization }\end{array}$ & $\begin{array}{l}\text { introduce BPSO gets from the way that, in rivalry with other meta-heuristics, } \\
\text { BPSO has demonstrated to be a champ by and large, putting a technique as a } \\
\text { genuine alternative when one needs to take care of a complex optimization }\end{array}$ \\
\hline Fuzzy logic & problem. The positioning is assessed utilizing fuzzy logic. Simulation \\
\hline Network security & Results on IEEE-14 and IEEE-30 bus systems are presented with different \\
\hline Power system optimization & objectives. \\
\hline
\end{tabular}

This is an open access article under the CC BY-SA license.

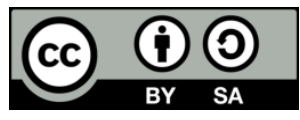

\section{Corresponding Author:}

Padmanabha Raju Chinda

Department of Electrical and Electronics Engineering, Prasad V. Potluri Siddhartha Institute of Technology Kanuru, Vijayawada, Andhra Pradesh-520007, India

Email: pnraju78@yahoo.com

\section{INTRODUCTION}

In serious electric power systems market, there is a need to work electric utilities closer as far as possible. To analyze whether a power system can stay in a protected and solid working state under contingency conditions, those possibility cases that will bring about loss of load, loss of generation, or islanding are first to be distinguished. In view of framework administrators past experience each post-unexpected amount is allotted a level of seriousness as indicated by potential harm that could be forced on the power system by the amount, should the contingency happens [1].

In the current day power system planning and operation considerable interest is being shown in contingency analysis. Possibility screening and positioning is one of the significant segments of on-line system security appraisal. The target of contingency screening and positioning is to rapidly and precisely select a short rundown of basic contingency from an enormous rundown of potential contingency and rank them as per their seriousness. Reasonable preventive control activities can be actualized considering contingency that are probably going to influence the power system execution. Milano et al. [2] proposed an OPF-based market clearing calculation that represented voltage security limits. The security compelled optimum-path forest (OPF) program limits the pre-possibility target work while watching both the pre-and post-possibility framework imperatives [3]. A tale way to deal with multi target particle swarm optimization strategy for taking care of ideal power flow issue was proposed in [4]. Anitha et al. [5] an full depth reclamation (FDR) particle swarm optimization (PSO) technique proposed to take care of the OPF issue considering incline rate cutoff points of generators and line flow limits. Transformative calculations have end 
up being ground-breaking meta-heuristic solvers when applied to complex issues [6]. Self-versatile models have demonstrated to improve the effectiveness of the calculations by and large. The self-versatile attributes are customarily given to calculations by controlling, under determination tension, the change rates utilized in the calculations.

PSO was enlivened by the social conduct of feathered creatures running and was proposed to take care of persistent issues. The first PSO is a ceaseless PSO (CPSO) being applied and reached out to take care of numerous nonstop issues [7]. PSO has likewise been reached out to adapt to discrete issues [8]. Binary particle swarm optimization (BPSO) was initially evolved by Kennedy and Eberhart [9], to take care of numerous combinatorial issues, for instance, workshop booking [10] and feature selection [11]. In BPSO, the position is a twofold vector, and the speed is a nonstop vector. BPSO has been applied to some genuine issues. Sarath and Ravi [12] applied BPSO to produce affiliation rules from value-based datasets. The outcomes on a dataset from an Indian business bank demonstrated that applying BPSO gave more excellent principles as well as dodged repetitive standards. Taha and Nadi [13] utilized BPSO to identify the accessible frequencies in cognitive radio, which permitted to use the framework assets.

The BPSO-based unique assignment accomplished higher recognition rates and lower bogus alert rates with various commotion proportions. Lin et al. [14] utilized BPSO to scan for exceptionally productive thing sets rather than visit thing sets in value-based databases. It was demonstrated that the BPSO-based calculation was increasingly proficient, progressively viable, and met quicker than GAs. In numerous different works, BPSO has likewise been changed to improve its presentation. In the first BPSO [15], a sigmoid function was utilized as an exchange work known as a shaped work. Mirjalili and Lewis [16], the V-formed transfer functions were proposed for BPSO. The position refreshing condition additionally thought about the past area. The trial results indicated that applying the V-molded functions while considering the past area improved the presentation of BPSO. Be that as it may, it was not satisfactory that which adjustment contributed more to the improvement. The exhibition of BPSO on various datasets intensely relied upon the particular transfer functions, in any event, when they were from a similar family (V-formed or S-molded). Consequently, it was anything but a simple undertaking to choose a proper transfer function for a specific errand or dataset. Islam et al. [17] proposed a period changing S-formed exchange work for BPSO which meant to give a smooth move from more investigation to more misuse during the developmental procedure. The trial results demonstrated that the time-changing transfer function helped BPSO to accomplish preferred outcomes over a standardized linear transfer function [18] and a notable V-molded transfer functions [16]. Liu et al. [19] gave a point by point investigation of the impact of the inertia weight parameter on the looking through capacity of BPSO.

The BPSO algorithm is extremely important for practical and commercial use in the resolution of discrete problems, so this algorithm helps optimize power systems networks. The BPSO improves communication between populations and maintains population diversity in order to overcome limitations of classical optimization algorithms in resolving multi-parameter, powerful coupling and nonlinear problem optimization in engineering. The BPSO algorithm is based on the traditional PSO algorithm to balance global search and local search by adjusting the inertial weight. In this paper multiple objectives based optimum-path forest (OPF) method using binary particle swarm optimization technique was discussed. Fuzzy based contingency ranking method was used to find most severe lines of contingencies. The effectiveness of the BPSO method in giving solution for various objectives was tested on IEEE 30-bus and IEEE 14-bus systems.

\section{METHODOLOGY FOR BINARY PARTICLE SWARM OPTIMIZATION}

In BPSO, every arrangement in the populace is a binary string. Every binary string is of measurement $\mathrm{n}$ which is assessed to give parameter esteems. In the BPSO, every binary string speaks to a molecule. Strings are refreshed a tiny bit at a time dependent on its present worth, the estimation of that bit in the best (fitness) of that molecule to date, and the best estimation of that bit to date of its neighbours [20]-[25]. For binary strings, neighbors can be chosen in one of a few different ways. A few models are: (for an area of size k). Neighbors are the k paired strings whose Hamming separation is limited. For equivalent Hamming separations, the decisions are subjective. In the first place, self-assertively dole out gatherings of $\mathrm{k}$ strings to neighborhoods. Let the local size be the populace size.

In standard PSO, everything is as far as a speed. By and large the speed is characterized as far as a likelihood of the bit evolving. In BPSO, a little bit at a time refresh is done probabilistically. At the end of the day, for a picked bit $\mathrm{d}$ in a picked string $\mathrm{I}$ it is changed to a 1 with a likelihood $\mathrm{P}$ that is an element of its inclination to be a 1 , the best estimation of itself to date, and the best estimation of its neighbors. (1-P) is the likelihood of changing to a zero. When $\mathrm{P}$ is resolved, an irregular number $\mathrm{R}$ is produced. On the off chance that $\mathrm{R}<\mathrm{P}$, at that point the bit turns into a 1 ; else it turns into a zero. 
Fundamentally, PSO and BPSO are the equivalent in the feeling of the consistent progression of the calculation, which in discovering pbest and gbest, update speed and position [8]. The main contrast among PSO and BPSO is the conditions used to characterize the updates of speed and position of every molecule. Equation (1) is utilized to refresh the speed while (2) and (3) are utilized to refresh the situation of every molecule.

$$
\begin{aligned}
& v_{i d}^{k+1}=w v_{i d}^{k}+c_{1} r_{1}\left(\text { pbest }_{i d}-x_{i d}\right)+c_{2} r_{2}\left(\text { gbest }_{i d}-x_{i d}\right) \\
& \operatorname{sigmoid}\left(v_{i d}^{k}\right)=\frac{1}{1+e^{-v_{i d}^{k}}} \\
& x_{i d}^{k}=\left\{\begin{array}{c}
1, \text { if rand }<\operatorname{sigmoid}\left(v_{i d}^{k}\right) \\
0, \text { otherwise }
\end{array}\right.
\end{aligned}
$$

The $\mathrm{x}_{\mathrm{i}}$ and velocity $\mathrm{v}_{\mathrm{i}}$ of an $\mathrm{i}$ particle are described by its position. When searches are carried out, each particle modifies its location in line with its own experience and experience of all its surrounding particles, using the best position it has $\left(\mathrm{p}_{\mathrm{i}}\right)$ and everyone else $\left(\mathrm{p}_{\mathrm{g}}\right)$.

\section{PROBLEM FORMULATION}

Objective function-1:

$$
F=\sum_{i-1}^{N G} F_{i}=\sum_{i-1}^{N G}\left(a_{i} P_{i}^{g^{2}}+b_{i} P_{i}^{g}+c_{i}\right) R s / h r
$$

where, $F_{i}$ is total cost function, $P_{i}^{g}$ is generation of active power (control variables), $N G$ is number of generator buses, $a_{i}, b_{i}, c_{i}$ : Coefficients of Fuel cost for $i^{\text {th }}$ unit.

Objective Function-2: Composite index based on line loadings, voltage profiles and voltage stability indices.

Composite logic criteria $(\mathrm{CLC})=\left(\mathrm{TI}_{\mathrm{LL}}+\mathrm{TI}_{\mathrm{VP}}+\mathrm{TI}_{\mathrm{VSI}}\right)$

CLC: Composite logic criteria

$\mathrm{TI}_{\mathrm{LL}}$ : Total index of line loadings

TIVP: Total index of voltage profiles

TIvs: Total index of voltage stability indices

Figure 1 depicts the process of computing composite logic criteria. In this data obtained from Newton-Raphson load flow solution (line loadings, load bus voltage profiles and voltage stability indices) will be given to the program of fuzzy inference. The fuzzy system segregates the input data based on the rules framed for fuzzification and forms the total index for line loadings, voltage profiles and voltage stability indices. By adding these three indices composite logic criteria will be formed. As per objective function-2, composite logic criteria will be minimized, which in turn improves the security of the system. If the security of the system was improved then overloading in the lines will be reduced, voltage profiles of the load buses will be improved. Fuzzy rules to calculate CLC was given in Table 1. LL: loaded lightly, LU: loaded usually, CL: completely loaded, LO: loaded over, LV: low voltage, NV: normal voltage, OV: over voltage, VLI: very low index, LI: low index, MI: medium index, HI: high index, VHI: very high index, LS: low severe, US: under severe, SA: severe above, HS: higher severity, VLS: very low severe.

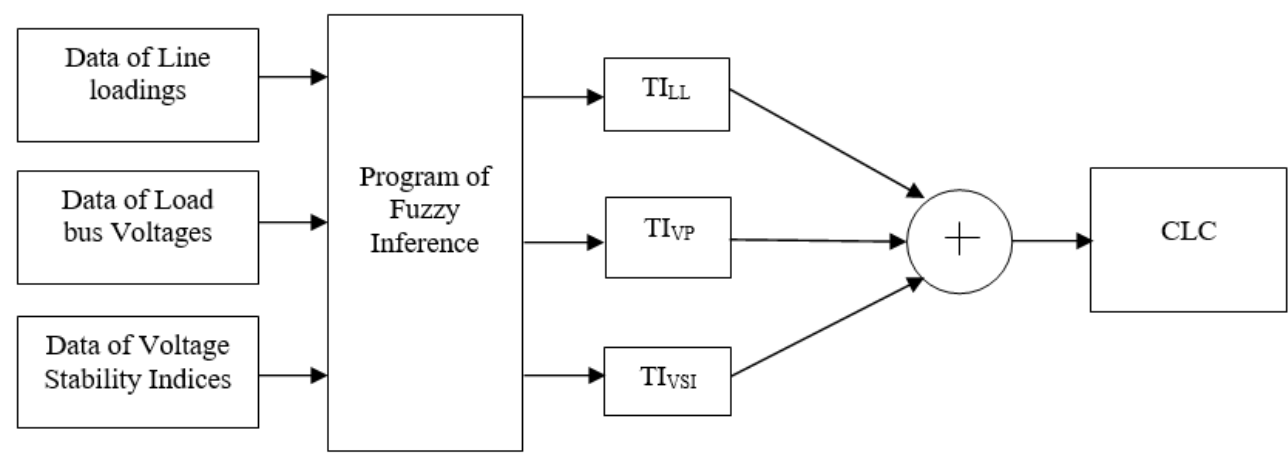

Figure 1. Process of calculating composite logic criteria 
Table 1. Fuzzy rule table

\begin{tabular}{|c|c|c|c|c|c|c|c|c|c|c|c|c|}
\hline \multicolumn{5}{|c|}{ Line Loadings } & \multicolumn{3}{|c|}{ Voltage Profiles } & \multicolumn{5}{|c|}{ Voltage stability Indices } \\
\hline Input & LL & $\mathrm{LU}$ & $\mathrm{CL}$ & LO & $\mathrm{LV}$ & NV & $\mathrm{OV}$ & VLI & LI & MI & $\mathrm{HI}$ & VHI \\
\hline Output & LS & US & SA & HS & US & SA & HS & VLS & LS & US & SA & HS \\
\hline
\end{tabular}

Above objective functions will be minimized subject to satisfaction of equality and inequality constraints. Newton-Raphson power flow methodology was used for power flow calculations. The equality constraint will be load balance equation i.e., total load and loss must be equal to total generation. The inequality constraints will be maintaining active power generation, reactive power generation, generator bus voltage profiles and load bus voltage profiles within their specified limits. Sets of non-linear flow of power equations, which regulate the system of power, are equality constraints.

$$
\begin{gathered}
P_{G i}-P_{D i}-\sum_{j=1}^{n}\left|V_{i}\right|\left|V_{j}\right| \| Y_{i j} \mid \cos \left(\theta_{i j}-\delta_{i}+\delta_{j}\right)=0 \\
Q_{G i}-Q_{D i}+\sum_{j=1}^{n}\left|V_{i}\right|\left|V_{j} \| Y_{i j}\right| \sin \left(\theta_{i j}-\delta_{i}+\delta_{j}\right)=0
\end{gathered}
$$

In cases where, $P_{G i}$ and $Q_{G i}$ are actual and reactive bus power outputs, load demand is represented by $P_{D i}$ and $Q_{D i}$ at the same bus, and the bus admittance matrix components of $\left|Y_{i j}\right|$ and $\theta_{i j}$ are represented.

Unequal treatment constraints: These are the limitations representing the operating system and its security limits as follows:

- Generators real and reactive power outputs

$$
\begin{aligned}
& P_{G i}^{\min } \leq P_{G i} \leq P_{G i}^{\max }, i=1, \ldots, N_{G} \\
& Q_{G i}^{\min } \leq Q_{G i} \leq Q_{G i}^{\max }, i=1 \ldots, N_{G}
\end{aligned}
$$

- Voltage magnitudes at each bus in the network

$$
V_{i}^{\min } \leq V_{i \leq} V_{i}^{\max }, i=1, \ldots . N L
$$

- Transformer tap settings

$$
T_{i}^{\min } \leq T_{i} \leq T_{i}^{\max }, i=1, \ldots N T
$$

- Reactive power injections due to capacitor banks

$$
Q_{C i}^{\min } \leq Q_{C i} Q_{C i}^{\max }, i=1, \ldots, C S
$$

- Transmission lines loading

$$
S_{i} \leq S_{i}^{\max }, i=1, \ldots, n l
$$

- Voltage stability index

$$
L j_{i} \leq L j_{i}^{\max }, i=1, \ldots, N L
$$

\section{FLOWCHART FOR IMPLEMENTATION OF BPSO}

Flowchart for the implementation of proposed methodology was shown in Figure 2. The main implementation steps as depicted in Figure 2 are given: i) initialize the multitude, the situation of particles is arbitrarily instated inside the hypercube of plausible space; ii) evaluate the exhibition of every molecule, utilizing its present position; iii) compare the presentation of every person to its best execution up until now; iv) compare the presentation of every molecule to the global best molecule; v) change the speed of the molecule; vi) move every molecule to another position; and vii) go to stage 2 , and rehash until convergence. 


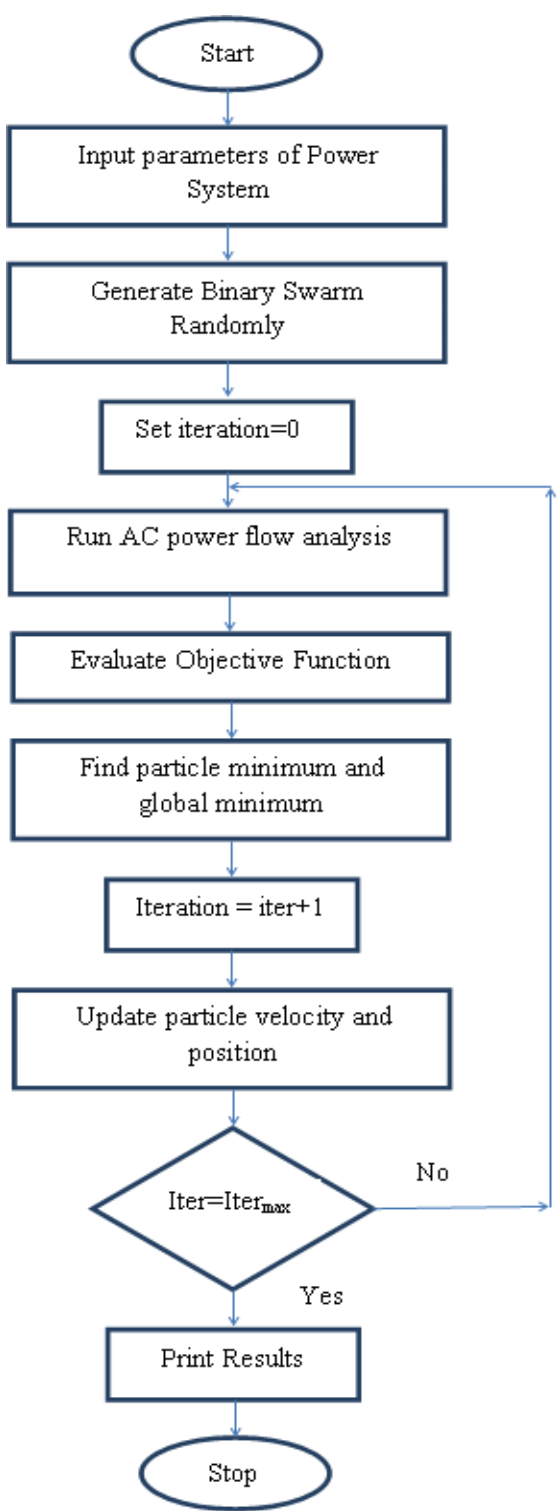

Figure 2. Flow chart for implementation of proposed methodology

\section{RESULTS AND DISCUSSION}

Test cases in this paper are carried out on modified IEEE 30-bus and IEEE 14-bus systems. For IEEE 30-bus system the outage of line connected between buses 2 and 5 was obtained as top-1 severe contingency. For IEEE 14-bus system after conducting contingency analysis line connected between buses 2 and 3 was observed as top-1 contingency. A comparison was made in between objective functions 1 and 2 and the obtained results were reported in Table 2 for IEEE 30 bus system and in Table 3 for IEEE 14 bus system. From these results it can be observed that BPSO was effective in achieving specified objective functions. Figures 3 and 4 depicts comparison of active power generations under two objective functions for IEEE 30 bus system and IEEE 14 bus systems respectively. Figures 5 and 6 show comparison of Generator Bus Voltage Profiles under two objective functions for IEEE 30 bus system and IEEE 14 bus systems respectively. From these figures it can be observed that control variables are within limits.

Table 2. Comparison of objective functions for IEEE 30 bus system

\begin{tabular}{ccc}
\hline Variable & Objective Function 1 & Objective Function 2 \\
\hline Fuel cost $(\$ / H r)$ & 828.4225 & 944.1078 \\
Composite index & 1590 & 1347 \\
Loss (p.u.MW) & 0.1392 & 0.0668 \\
Computation time $(\mathrm{sec})$ & 219.7030 & 371.61 \\
\hline
\end{tabular}

A binary particle swarm optimization approach for power system security ... (Padmanabha Raju Chinda) 
Table 3. Comparison of objective functions for IEEE 14 bus system

\begin{tabular}{ccc}
\hline Variable & Objective Function 1 & Objective Function 2 \\
\hline Fuel cost $(\$ / \mathrm{Hr})$ & 857.0573 & 1085.4 \\
Composite index & 1016 & 957 \\
Loss (p.u.MW) & 0.0867 & 0.0268 \\
Computation time (sec) & 77.797 & 118.109 \\
\hline
\end{tabular}

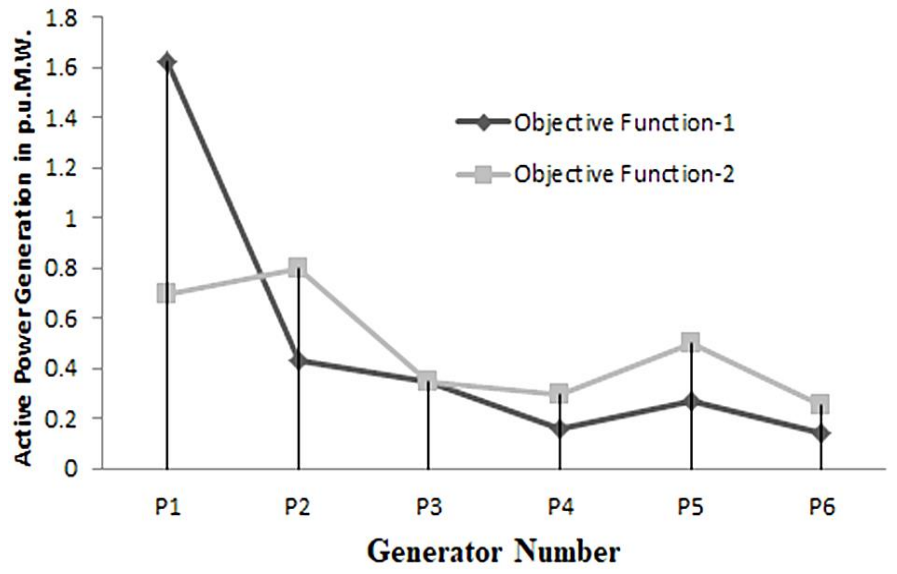

Figure 3. Comparison of active power generations under two objective functions for IEEE 30 bus system

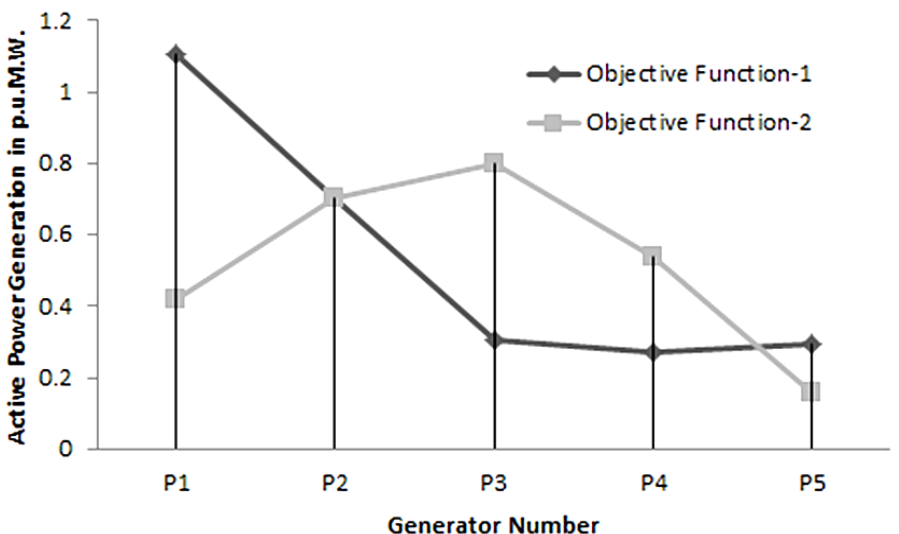

Figure 4. Comparison of active power generations under two objective functions for IEEE 14 bus system

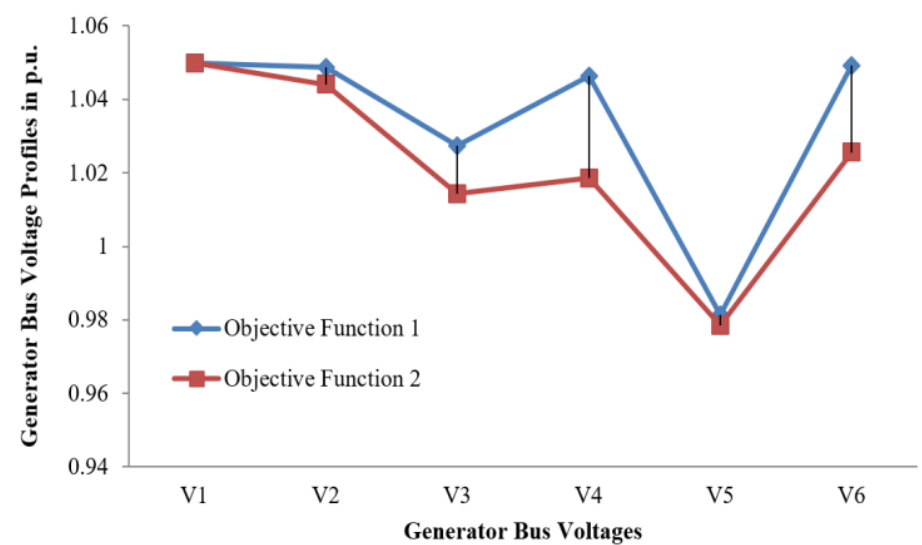

Figure 5. Comparison of generator bus voltage profiles under two objective functions for IEEE 30 bus system 


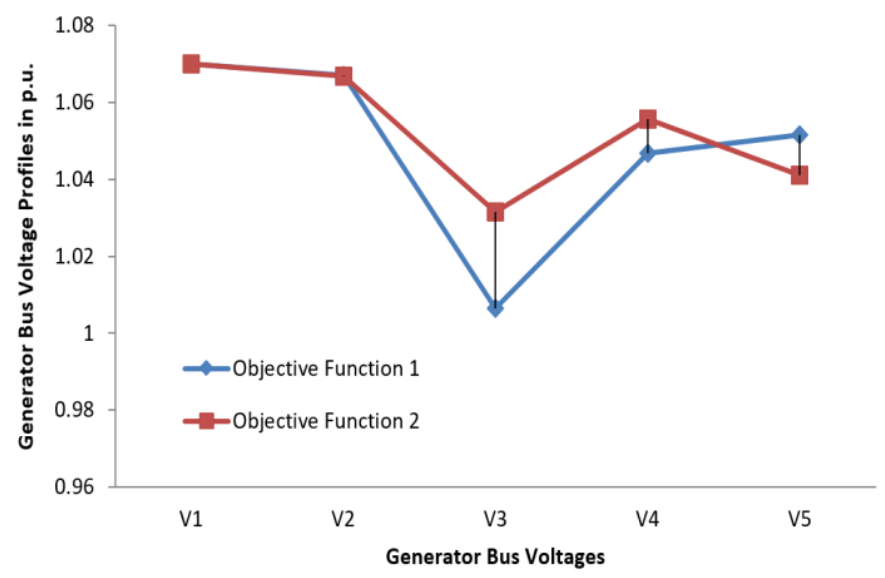

Figure 6. Comparison of generator bus voltage profiles under two objective functions for IEEE 14 bus system

\section{CONCLUSION}

From these results on the IEEE 30-bus and IEEE 14-bus systems, it can be seen that numerical results demonstrate the feasibility as well as the effectiveness of the OPF method. By generation rescheduling using proposed BPSO-OPF the security of the system can be improved effectively. This BPSO-OPF method is useful for providing an efficient solution for multiple objective functions. BPSO based OPF will be a very useful tool to operate and manage the electric power systems in efficient ways and it can be used for optimization in practical power systems. The results clearly indicates that the OPF method works satisfactorily in bringing a superior solution for the specified objective function while maintaining all control variables within limits.

\section{REFERENCES}

[1] Y.-Y. Hsu and H.-C. Kuo, "Fuzzy-set based contingency ranking (power system security)," IEEE Transactions on Power Systems, vol. 7, no. 3, pp. 1189-1196, 1992, doi: 10.1109/59.207333.

[2] F. Milano, C. A. Canizares, and A. J. Conejo, "Sensitivity-based security-constrained OPF market clearing model," IEEE Transactions on Power Systems, vol. 20, no. 4, pp. 2051-2060, Nov. 2005, doi: 10.1109/TPWRS.2005.856985.

[3] R. C. Degeneff, W. Neugebauer, C. H. Saylor, and S. L. Corey, "Security constrained optimization: an added dimension in utility systems optimal power flow," IEEE Computer Applications in Power, vol. 1, no. 4, pp. 26-30, Oct. 1988, doi: 10.1109/67.20549.

[4] M. A. Abido, "Multiobjective particle swarm optimization for optimal power flow problem," in 2008 12th International MiddleEast Power System Conference, Mar. 2008, pp. 392-396, doi: 10.1109/MEPCON.2008.4562380.

[5] M. Anitha, S. Subramanian, and R. Gnanadass, "FDR PSO-based transient stability constrained optimal power flow solution for deregulated power industry," Electric Power Components and Systems, vol. 35, no. 11, pp. 1219-1232, Aug. 2007, doi: $10.1080 / 15325000701351641$

[6] A. AL Ahmad and R. Sirjani, "Optimal placement and sizing of multi-type FACTS devices in power systems using metaheuristic optimisation techniques: An updated review," Ain Shams Engineering Journal, vol. 11, no. 3, pp. 611-628, Sep. 2020, doi: 10.1016/j.asej.2019.10.013.

[7] Y.-J. Gong et al., "Genetic learning particle swarm optimization," IEEE Transactions on Cybernetics, vol. 46, no. 10, pp. 2277-2290, Oct. 2016, doi: 10.1109/TCYB.2015.2475174.

[8] W.-N. Chen, J. Zhang, H. S. H. Chung, W.-L. Zhong, W.-G. Wu, and Y.-h. Shi, "A novel set-based particle swarm optimization method for discrete optimization problems," IEEE Transactions on Evolutionary Computation, vol. 14, no. 2, pp. 278-300, Apr. 2010, doi: 10.1109/TEVC.2009.2030331.

[9] J. Kennedy and R. Eberhart, "Particle swarm optimization," in Proceedings of ICNN'95 - International Conference on Neural Networks, vol. 4, pp. 1942-1948, doi: 10.1109/ICNN.1995.488968.

[10] M. Eddaly, B. Jarboui, and P. Siarry, "Combinatorial particle swarm optimization for solving blocking flowshop scheduling problem," Journal of Computational Design and Engineering, vol. 3, no. 4, pp. 295-311, 2016, doi: 10.1016/j.jcde.2016.05.001.

[11] H. Banka and S. Dara, "A Hamming distance based binary particle swarm optimization (HDBPSO) algorithm for high dimensional feature selection, classification and validation,” Pattern Recognition Letters, vol. 52, pp. 94-100, Jan. 2015, doi: 10.1016/j.patrec.2014.10.007.

[12] K. N. V. D. Sarath and V. Ravi, “Association rule mining using binary particle swarm optimization," Engineering Applications of Artificial Intelligence, vol. 26, no. 8, pp. 1832-1840, Sep. 2013, doi: 10.1016/j.engappai.2013.06.003.

[13] M. A. Taha and D. I. Abu al Nadi, "Spectrum sensing for cognitive radio using binary particle swarm optimization," Wireless Personal Communications, vol. 72, no. 4, pp. 2143-2153, Oct. 2013, doi: 10.1007/s11277-013-1140-x.

[14] J. C.-W. Lin, L. Yang, P. Fournier-Viger, T.-P. Hong, and M. Voznak, "A binary PSO approach to mine high-utility itemsets," Soft Computing, vol. 21, no. 17, pp. 5103-5121, Sep. 2017, doi: 10.1007/s00500-016-2106-1.

[15] R. Poli, J. Kennedy, and T. Blackwell, "Particle swarm optimization," Swarm Intelligence, vol. 1, no. 1, pp. 33-57, Oct. 2007, doi: 10.1007/s11721-007-0002-0.

[16] S. Mirjalili and A. Lewis, "S-shaped versus V-shaped transfer functions for binary particle swarm optimization," Swarm and Evolutionary Computation, vol. 9, pp. 1-14, Apr. 2013, doi: 10.1016/j.swevo.2012.09.002.

[17] M. J. Islam, X. Li, and Y. Mei, "A time-varying transfer function for balancing the exploration and exploitation ability of a binary

A binary particle swarm optimization approach for power system security ... (Padmanabha Raju Chinda) 
PSO,” Applied Soft Computing, vol. 59, pp. 182-196, Oct. 2017, doi: 10.1016/j.asoc.2017.04.050.

[18] J. C. Bansal and K. Deep, "A modified binary particle swarm optimization for knapsack problems," Applied Mathematics and Computation, vol. 218, no. 22, pp. 11042-11061, Jul. 2012, doi: 10.1016/j.amc.2012.05.001.

[19] J. Liu, Y. Mei, and X. Li, "An analysis of the inertia weight parameter for binary particle swarm optimization," IEEE Transactions on Evolutionary Computation, vol. 20, no. 5, pp. 666-681, Oct. 2016, doi: 10.1109/TEVC.2015.2503422.

[20] R. F. Malik and H. Hermawan, "Credit scoring using classification and regression tree (CART) algorithm and binary particle swarm optimization," International Journal of Electrical and Computer Engineering (IJECE), vol. 8, no. 6, pp. 5425-5431, Dec. 2018, doi: 10.11591/ijece.v8i6.pp5425-5431.

[21] M. Kannan, K. Sampath, S. Pattabiraman, K. Narayanan, and T. Senjyu, "Meta-heuristic BPSO based voltage profile enhancement in radial distribution system through network reconfiguration," International Journal of Emerging Electric Power Systems, vol. 20, no. 6, Dec. 2019, doi: 10.1515/ijeeps-2019-0110.

[22] D. Wang, D. Tan, and L. Liu, "Particle swarm optimization algorithm: an overview," Soft Computing, vol. 22, no. 2, pp. 387-408, Jan. 2018, doi: 10.1007/s00500-016-2474-6.

[23] E. Lu, L. Xu, Y. Li, Z. Ma, Z. Tang, and C. Luo, "A novel particle swarm optimization with improved learning strategies and its application to vehicle path planning," Mathematical Problems in Engineering, vol. 2019, pp. 1-16, Nov. 2019, doi: $10.1155 / 2019 / 9367093$

[24] M. A. Tawhid and K. B. Dsouza, "Hybrid binary bat enhanced particle swarm optimization algorithm for solving feature selection problems," Applied Computing and Informatics, vol. 16, no. 1/2, pp. 117-136, Apr. 2018, doi: 10.1016/j.aci.2018.04.001.

[25] A. N. Hussain and A. A. Ismail, "Operation cost reduction in unit commitment problem using improved quantum binary PSO algorithm," International Journal of Electrical and Computer Engineering (IJECE), vol. 10, no. 2, pp. 1149-1155, Apr. 2020, doi: 10.11591/ijece.v10i2.pp1149-1155.

\section{BIOGRAPHIES OF AUTHORS}
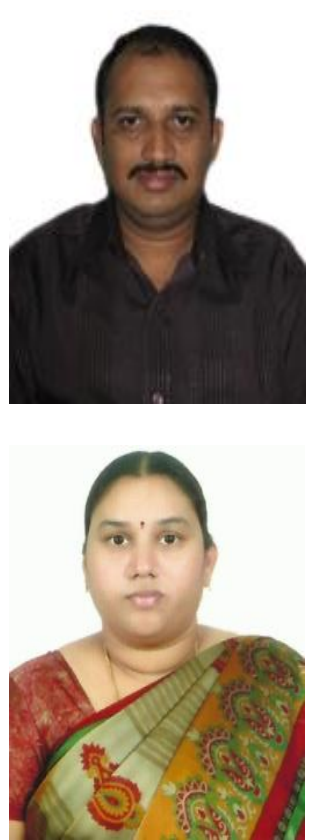

Padmanabha Raju Chinda (D) S SC P is currently working as Professor in the Department of Electrical and Electronics Engineering, P.V.P. Siddhartha Institute of Technology, Vijayawada, Andhra Pradesh, India. He obtained Ph.D. from J.N.T. University, Kakinada. His areas of interest are Power System Security, OPF techniques, Deregulation, FACTS and Smart Grid. He can be contacted at email: pnraju78@yahoo.com.

Ragaleela Dalapati Rao (D) SI SC P is currently working as Senior Assistant Professor in the EEE department of P.V.P. Siddhartha Institute of Technology, Vijayawada. She completed her graduation from J.N.T. University, Hyderabad and did M Tech with Power Systems specialization from Acharya Nagarjuna University, Guntur. Presently pursuing Ph.D. at JNTUK, Kakinada. Her areas of interest are OPF, FACTS and Power system deregulation. She can be contacted at email: raga_233@yahoo.co.in. 